\title{
SHAPE FUNCTIONS OF DIPOLAR FERROMAGNETS AT THE CURIE POINT
}

\author{
E. FREY, F. SCHWABL and S. THOMA \\ Institut für Theoretische Physik, Physik-Department der Technischen Universität München, D-8046 Garching, FRG
}

Received 18 February 1988; accepted for publication 25 March 1988

Communicated by A.A. Maradudin

\begin{abstract}
We present a complete mode coupling theory for the critical dynamics of ferromagnets above the Curie point with both short range exchange and long range dipolar interaction. This theory allows us to determine the full Kubo relaxation functions at the critical point. In particular, we are able to explain recent spin echo measurements.
\end{abstract}

An increasing quantitative understanding of the critical dynamics of isotropic ferromagnets such as EuO, EuS and Fe above $T_{\mathrm{c}}$ has been achieved recently by taking into account the long range dipolar interaction, which is present in all real ferromagnets. The results of various experimental methods [1-11] (neutron scattering, electron spin resonance and hyperfine interaction), sampling different regions in $q$-space, could be explained in a unified fashion within a mode coupling (MC) theory $[12,13]$. Therefore dipolar interactions are seen to be highly relevant for the description of the critical dynamics of real ferromagnets.

The standard procedure of MC theory usually incorporates two approximations. The first is to consider only two-mode decay processes, which amounts to a factorisation approximation of the Kubo relaxation functions after insertion of the microscopic equations of motion. The second step is a lorentzian approximation for the Kubo relaxation functions of the spin variables $S^{i}(q, t)$, which are defined by

$\boldsymbol{\Phi}^{i j}(\boldsymbol{q}, t)=\mathrm{i} \lim _{\epsilon \rightarrow 0} \int_{1}^{\infty} \mathrm{d} \tau \mathrm{e}^{-\epsilon \tau}\left\langle\left[S^{i}(\boldsymbol{q}, \tau), S^{j}(\boldsymbol{q}, 0)^{\dagger}\right\rangle\right.$,

with the normalization $\Phi^{i j}(q, t=0)=1$; i.e. the spin variables are normalized with respect to the static susceptibilities. The corresponding frequency dependent functions are defined by a half sided Fourier transform

$\boldsymbol{\Phi}^{i j}(\boldsymbol{q}, \omega)=\int_{0}^{\infty} \mathrm{d} t \mathrm{e}^{i \omega t} \Phi^{i j}(\boldsymbol{q}, t)$.

The lorentzian approximation gives for most purposes an excellent approximation for the linewidth as can be inferred from refs. [12] and [13]. However, in order to obtain information about the lineshape one has to refrain from this approximation. This has been achieved for the case of pure exchange interaction by Hubbard [14] and Wegner [15], using a discrete version of the MC theory. A simplified version of the coupled mode equations has been given recently by Lovesey and Williams [16] and Balucani et al. [17]. Besides these $M C$ theories there exist renormalization group ( $R G$ ) calculations for isotropic ferromagnets [18-22]. All these theories, taking into account only the short range exchange interaction, are unable to explain the nearly exponential decay of the transverse relaxation function for small times, found in spin echo measurements on EuO [23]. This led Balucani et al. to the conclusion that neither MC theory nor RG theory is valid in the time regime probed in Mezei's experiments. To fit the experimental results they proposed a "hybrid theory", which is a phenomenological interpolation scheme between the short and long time limits. In constrast we will show 
in this Letter that the experiment can be explained naturally within our MC theory by including dipolar forces without any need for a special treatment of the short time behaviour.

The hamiltonian for a spin system including both short range exchange and long range dipolar interactions is given by [24]

$H=\int \frac{\mathrm{d}^{3} q}{(2 \pi)^{3}}\left[\left(J_{0}+J q^{2}\right) \delta^{i j}+J g \frac{q^{i} q^{j}}{q^{2}}\right] S^{i}(\boldsymbol{q}) S^{j}(-\boldsymbol{q})$,

where $S^{i}(\boldsymbol{q})=\int \mathrm{d}^{3} x \mathrm{e}^{\mathrm{i} \boldsymbol{q} \cdot \boldsymbol{x}} S^{i}(\boldsymbol{x})$ are the Fourier transforms of the cartesian components of the spin operator $S^{i}(\boldsymbol{x})$. The parameter $g=a_{1}\left(g_{\mathrm{L}} \mu_{\mathrm{B}}\right)^{2} / 2 \mathrm{Ja}^{3}=\left(q_{\mathrm{D}} a\right)^{2}$ characterizes the ratio of the dipolar to the exchange interaction $J$. Here $g_{L}$ is the Landé factor and the coefficient $a_{1}$ depends on the latice structure; i.e.: $a_{1}=4 \pi(\mathrm{sc}), 3^{3 / 2} \pi$ (bcc), $2^{5 / 2} \pi$ (fcc). The parameter $J_{0}$ does not enter the equations of motion. In eq. (1) we assumed that the dipolar forces are weaker than the exchange interaction [24]; i.e. $g \ll 1$. All quantities are given in units of the lattice spacing $a$.

The symmetry of the hamiltonian suggests decomposing the spin operator $S(q)$ into one longitudinal and two transverse components with respect to the wavevector $\boldsymbol{q}$; i.e. $\boldsymbol{S}(\boldsymbol{q})=S^{\mathrm{L}}(\boldsymbol{q}) \hat{\boldsymbol{q}}+S^{\mathrm{T}_{1}}(\boldsymbol{q}) \hat{\boldsymbol{t}}^{1}(\hat{\boldsymbol{q}})+S^{\mathrm{T}_{2}}(\boldsymbol{q}) \hat{\boldsymbol{t}}^{2}(\hat{\boldsymbol{q}})$, where the orthonormal set of unit vectors is defined by $\hat{\boldsymbol{q}}=\boldsymbol{q} / q, \hat{\boldsymbol{t}}^{1}(\boldsymbol{\boldsymbol { q }})=\boldsymbol{q} \times \boldsymbol{e}_{3} / \sqrt{q_{1}^{2}+q_{2}^{2}}, \hat{\boldsymbol{t}}^{2}(\hat{\boldsymbol{q}})=\hat{\boldsymbol{q}} \times \hat{\boldsymbol{t}}^{1}(\hat{\boldsymbol{q}})$. For vanishing components of $q$ the limits are taken in the order of increasing cartesian components.

The resulting equations of motion can be found in ref. [12]. From these one obtains by the first step of MC theory the following set of coupled integro-differential equations for the Kubo relaxation fucntions $\Phi^{\alpha}(q, g$, $t)$ and the transport coefficients $\Gamma^{\alpha}(q, g, t)(\alpha=\mathrm{L}, \mathrm{T})$ for the longitudinal and transverse modes (with respect to the wave vector $q$ ) [13]:

$\frac{\partial}{\partial t} \Phi^{\alpha}(q, g, t)=-\int_{0}^{t} \mathrm{~d} \tau \Gamma^{\alpha}(q, g, t-\tau) \Phi^{\alpha}(q, g, \tau)$.

The transport coefficients $\Gamma^{\alpha}(q, g, t)$ are related to the relaxation functions $\Phi^{\alpha}(q, g, t)$ by

$$
\begin{gathered}
\Gamma^{\alpha}(q, g, t)=4 J^{2} k_{\mathrm{B}} T \int_{\mathrm{BZ}} \frac{\mathrm{d}^{3} k}{(2 \pi)^{3}} \sum_{\beta, \sigma} v_{\beta \sigma}^{\alpha}(k, q, g, \theta)\left(\delta^{\sigma . \mathrm{T}}+\delta^{\alpha . \mathrm{T}} \sigma^{\beta, \mathrm{L}} \delta^{\sigma, \mathrm{L}}\right) \\
\times \frac{\chi^{\beta}(k, g) \chi^{\sigma}(|\boldsymbol{q}-\boldsymbol{k}|, q)}{\chi^{\alpha}(q, g)} \Phi^{\beta}(k, g, t) \Phi^{\sigma}(|\boldsymbol{q}-\boldsymbol{k}|, g, t),
\end{gathered}
$$

where the $k$-space integration runs over the first Brillouin zone (BZ). The vertex functions $v_{\beta \sigma}^{\alpha}$ for the decay of the mode $\alpha$ into the modes $\beta$ and $\sigma$ can be found in refs. $[12,13]$ and $\chi^{\alpha}(q, g)$ denote the static susceptibilities.

Now the essential point is that the MC equations (2) and (3) are consistent with the generalised dynamical scaling laws

$\Gamma^{\alpha}(q, g, \omega)=\Lambda q^{z} \Omega^{\alpha}(x, y) \gamma^{\alpha}\left(x, y, \nu_{\alpha}\right), \quad \Phi^{\alpha}(q, g, \omega)=\frac{1}{A q^{z} \Omega^{\alpha}(x, y)} \phi^{\alpha}\left(x, y, \nu_{\alpha}\right)$,

where in the present case we have to introduce the scaling variables

$x=\frac{1}{q \xi}, \quad y=\frac{\sqrt{g}}{q}$ and $\quad \nu_{\alpha}=\frac{\omega}{\Lambda q^{2} \Omega^{\alpha}(x, y)}$.

The scaled frequencies contain the characteristic frequencies $\Omega^{\alpha}(x, y)$. This implies for the Laplace transformed quantities the scaling laws

$\Phi^{\alpha}(q, g, t)=\phi^{\alpha}\left(x, y, \tau_{\alpha}\right), \quad \Gamma^{\alpha}(q, g, t)=\left[\Lambda q^{z} \Omega^{\alpha}(x, y)\right]^{2} \gamma^{\alpha}\left(x, y, \tau_{\alpha}\right)$,

where the scaled time variable $\tau_{\alpha}$ is given by 
$\tau_{\alpha}=\Lambda q^{z} \Omega^{\alpha}(x, y) t$

Inserting eq. (6) together with the static scaling law $\chi^{\alpha}(q, g)=\left(1 / J q^{2}\right) \hat{\chi}^{\alpha}(x, y)$ into eqs. (2) and (3) we find for the dynamic scaling functions

$$
\begin{aligned}
& \gamma^{\alpha}\left(x, y, \tau_{\alpha}\right)=2\left(\frac{\pi}{\Omega^{\alpha}(x, y)}\right)^{2} \int_{-1}^{1} \mathrm{~d} \eta \int_{0}^{\rho_{\mathrm{cut}}} \mathrm{d} \rho \rho_{-}^{-2} \sum_{\beta, \sigma} \hat{v}_{\beta \sigma}^{\alpha}(y, \rho, \eta)\left(\delta^{\sigma, \mathrm{T}}+\delta^{\alpha, \mathrm{T}} \delta^{\beta, \mathrm{L}} \delta^{\sigma, \mathrm{L}}\right) \\
& \frac{\hat{\chi}^{\beta}(x / \rho, y / \rho) \hat{\chi}^{\sigma}\left(x / \rho_{-}, y / \rho_{-}\right)}{\hat{\chi}^{\alpha}(x, y)} \phi^{\beta}\left(x / \rho, y / \rho, \tau_{\alpha \beta}(x, y, \rho)\right) \phi^{\sigma}\left(x / \rho_{-}, y / \rho_{-}, \tau_{\alpha \sigma}\left(x, y, \rho_{-}\right)\right)
\end{aligned}
$$

and the integro-differential equations

$\frac{\partial}{\partial \tau_{\alpha}} \phi^{\alpha}\left(x, y, \tau_{\alpha}\right)=-\int_{0}^{\tau_{\alpha}} \mathrm{d} \tau \gamma^{\alpha}\left(x, y, \tau_{\alpha}-\tau\right) \phi^{\alpha}(x, y, \tau)$,

which connect the scaling functions for the transport coefficients with the scaling functions for the Kubo relaxation functions. In eq. (8) we used the notations $\rho=k / q, \rho_{-}=|\boldsymbol{q}-\boldsymbol{k}| / q, \eta=\cos (\boldsymbol{q}, \boldsymbol{k})$ and $\tau_{\alpha \beta}(x, y, \lambda)=$ $\tau_{\alpha} \lambda=\Omega^{\beta}(x / \lambda, y / \lambda) / \Omega^{\alpha}(x, y)$.

The non-universal frequency scale of eq. (4) is found to be

$\Lambda=a^{5 / 2} \sqrt{J k_{\mathrm{B}} T / 2 \pi^{4}}=\frac{g_{\mathrm{L}} \mu_{\mathrm{B}}}{q_{\mathrm{D}}} \sqrt{k_{\mathrm{B}} T a_{1} / 4 \pi^{4}}$.

The above relations contain the critical dynamic exponent $z=5 / 2$, where one has to realize that the crossover of the critical dynamic exponent is contained in the scaling functions for the transport coefficients $\gamma^{\alpha}\left(x, y, \tau_{\alpha}\right)$, the scaling functions for the Kubo relaxation functions $\phi^{\alpha}\left(x, y, \tau_{\alpha}\right)$ and the characteristic frequencies $\Omega^{\alpha}(x$, $y)$. The scaled vertex function $\hat{v}_{\beta \sigma}^{\alpha}(y, \rho, \eta)$ can be found in refs. [12,13].

Due to the fact that the $k$-space integration is bounded by the Brillouin zone there is a cutoff in the $\rho$-integration of eq. (8), which is given by $\rho_{\text {cut }}=q_{\mathrm{Bz}} / q=\left(q_{\mathrm{Bz}} / q_{\mathrm{D}}\right) y$, where $q_{\mathrm{Bz}}$ denotes the boundary of the first Brillouin zero and $q_{\mathrm{D}}$ is the static crossover wavevector. This cutoff is important for small times, because the integrand of eq. (8) is proportional to 1 for $t=0$ and $\rho \gg 1$. Hence for small times wavevectors near the zone boundary are expected to be important for the relaxation mechanism. These non-universal modes are included in the above $\mathrm{MC}$ equations.

In order to incorporate the leading crossover behaviour into the time scale $\tau_{\alpha}$ it is convenient to choose the characteristic frequencies $\Omega^{\alpha}(x, y)$ as the linewidths resulting from the MC equations including the lorentzian approximation $\Omega^{\alpha}(x, y)=\gamma_{\text {lor }}^{\alpha}(x, y)$, where the scaling functions for the lorentzian linewidths $\gamma_{\text {lor }}^{\alpha}(x, y)$ are determined by a set of coupled integral equations (see eq. (10) in ref. [12]).

For the static susceptibilities we will use in the numerical calculations the Ornstein-Zernike forms

$\chi^{\alpha}(q, g)=\frac{1}{J\left(q^{2}+\xi^{-2}+\delta^{\alpha, L} g\right)}$,

where we note that MC theory does not account for effects of the critical exponent $\eta$, which will be neglected in the following. Here $\left.\xi=\xi_{0}\left[\left(T-T_{\mathrm{c}}\right) / T_{\mathrm{c}}\right)\right]^{-\nu}$ is the correlation length, which contains the static crossover through the effective exponent $\nu \approx \gamma_{\text {eff }} / 2$ [25].

Using a self-consistent numerical procedure we have solved the set of coupled integro-differential equations (8) and (9) at the critical temperature $(x=0)$, where we have used the values $q_{\mathrm{D}}=0.145 \AA^{-1}$ and $q_{\mathrm{Bz}}=1.06$ $\AA^{-1}$ corresponding to EuO from ref. [23]. The transverse and longitudinal scaling functions $\phi^{\alpha}\left(x=0, y, \tau_{\alpha}\right)$ versus the scaling variables $\tau_{\alpha}$ and $y=q_{\mathrm{D}} / q$ are shown in figs. 1 and 2 . The characteristic frequencies $\gamma_{\text {lor }}^{\alpha}(x, y)$ versus $y^{-1}$ can be found in fig. 7 of ref. [13]. The lineshapes for the longitudinal and transverse Kubo 


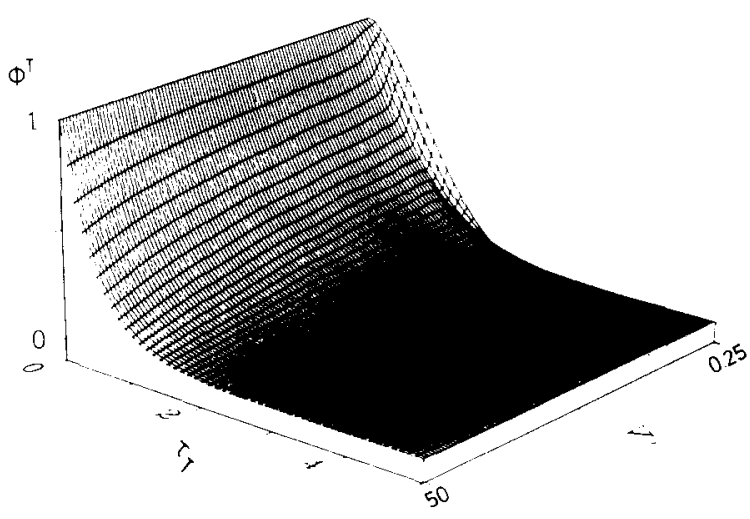

Fig. 1. Scaling function for the transverse Kubo relaxation function $\phi^{\mathrm{T}}\left(x=0, y, \tau_{\mathrm{T}}\right)$ versus $y=q_{\mathrm{D}} / q$ and $\tau_{\mathrm{T}}$ at $T=T_{c}$

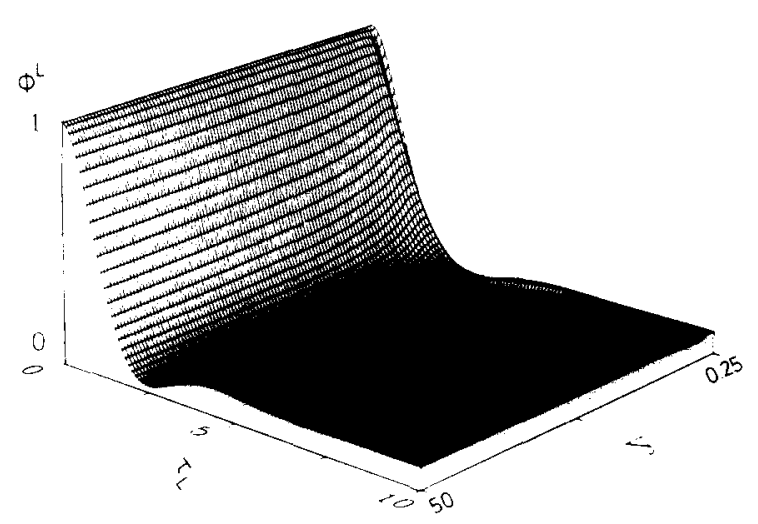

Fig. 2. Scaling function for the longitudinal Kubo relaxation function $\phi^{\mathrm{L}}\left(x=0, y, \tau_{\mathrm{L}}\right)$ versus $y=q_{\mathrm{D}} / q$ and $\tau_{\mathrm{L}}$ at $T=T_{c}$.

relaxation function differ significantly, especially for small wavevectors $q \ll q_{\mathrm{D}}$; i.e. $y \gg 1$. Both functions drop off quadratically at $t=0$, as is implied by eq. (9), but the transverse relaxation function obeys this behaviour in a very small time domain only and may be approximated best by an exponential function for $y \gg 1$. The longitudinal relaxation function shows a pronounced oscillatory behaviour.

Now we compare our theoretical results with experimental data starting with the spin echo measurements of Mezei [23] on EuO. Taking a cut of the transverse Kubo relaxation function in fig. 1 at the wavevector $q=0.024 \AA^{-1}$ we find the relaxation function shown in fig. 3 as the solid line versus time in $\mathrm{ns}$, where we have used the theoretical value for the non-universal scale $A=7.1 / 5.1326 \mathrm{meV} \AA^{5 / 2}$. There is an excellent agreement with the experimental data for $t \leqslant 1 \mathrm{~ns}$. The experimental data are above the theoretical curve for $t \geqslant 1 \mathrm{~ns}$. This may be due to finite collimation effect in this time domain, as noted by Mezei [23]. To substantiate this point we have also plotted in fig. 3 the relaxation function at $q=0.028 \AA^{-1}$ (dash-dotted curve), which is significantly higher than the curve for $q=0.024 \AA^{-1}$ for $t \geqslant 1$ ns. The fairly large difference of the curves with $q=0.024 \AA^{-1}$ and $q=0.028 \AA^{-1}$ comes from the vicinity of the crossover region.

In order to exhibit the difference from the MC theory including only short range exchange interaction, we

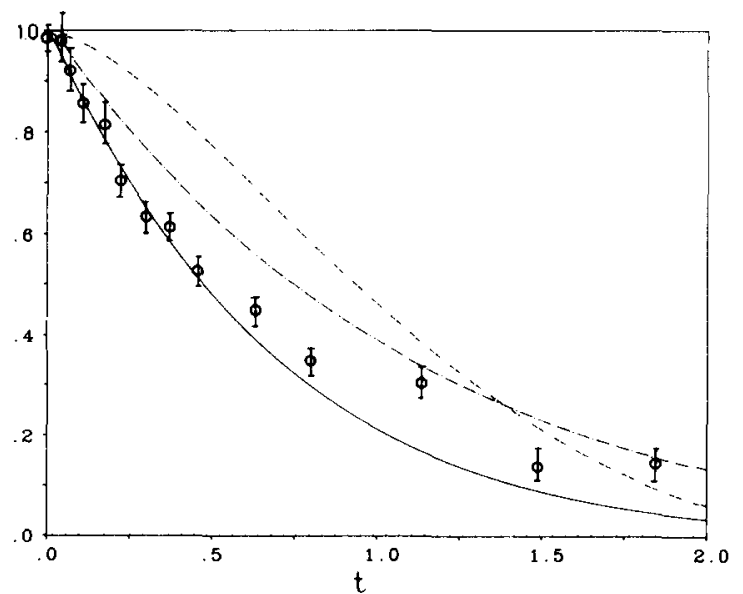

Fig. 3. Transverse Kubo relaxation function $\Phi^{\top}(q, g, t)$ at $q=0.024 \AA^{-1}$ (solid line) and $q=0.028 \AA^{-1}$ (dash-dotted line) for dipolar ferromagnets versus time $t$ in $n s$. The dashed line is the transverse Kubo relaxation function for short range exchange interaction only at $q=0.024 \AA^{-1}$. Data points from fig. 1 of ref. [23]. 
have solved eqs. (8) and (9) for this special case; i.e. $y=0, x=0$ and $\rho_{\text {cut }}=q_{\mathrm{Bz}} / q$ with $q=0.024 \AA^{-1}$. The result is the dashed curve in fig. 3 , which differs drastically from the lineshape including the long range dipolar interaction. So we conclude that the dipolar interaction is essential for the lineshape in the critical region. It is important to realize that the crossover in the lineshape starts nearly at $q_{\mathrm{D}}$, whereas the linewidth still scales with the isotropic critical dynamic exponent $z=5 / 2$ in this wavevector region.

In conventional neutron scattering one measures the scattering function $S(q, \omega)$

$S(q, \omega) \propto 2 k_{\mathrm{B}} T q^{-2} \hat{\chi}^{\mathrm{T}}(x, y) F(q, \omega) \frac{\omega / k_{\mathrm{B}} T}{1-\mathrm{e}^{-\omega / k_{\mathrm{B}} \mathrm{T}}}$,

where the spectral weight function $F(q, \omega)$ is related to the real part of the transverse Kubo relaxation functions by

$F(q, \omega)=\operatorname{Re}\left(\Phi^{\mathrm{T}}(q, \omega)\right)=\frac{1}{\Lambda q^{z} \Omega^{\mathrm{T}}(x, y)} \operatorname{Re}\left(\phi^{\mathrm{T}}\left(q, \nu_{\mathrm{T}}\right)\right)$.

The real parts of the transverse and longitudinal scaling functions for the Kubo relaxation functions $\phi^{\alpha}(x=0$, $\left.y, \nu_{\alpha}\right)$ are given in figs. 4 and 5 versus $y$ and the scaled frequency variables $\nu_{\alpha}$. A sensitive tool for the determination of the lineshape are constant energy scans for the scattering functions $S(q, \omega)$. Taking the theoretical value for the non-universal constant $\Lambda=7.1 / 5.1326 \mathrm{meV}^{5 / 2}$ we have calculated constant energy scans for a set of energies: $E=0.1 \mathrm{meV}, 0.2 \mathrm{meV}$ and $0.3 \mathrm{meV}$. The results are shown as the solid lines in fig. 6 . Comparing these results with experimental constant $E$ scans at $E=0.2 \mathrm{meV}$ and $E=0.3 \mathrm{meV}$ [26] we find a good agreement of the peak positions as well as the shape of the scans (compare fig. 3 of ref. [26]). For a quantitative comparison the theory has to be convoluted with the instrumental resolution function. We note that RG calculations [18-22], which include only exchange forces, account for the experimental data equally well as our MC theory in the energy domain probed by the experiments. This leads us to the conclusion that there is no significant influence of the dipolar forces on constant energy scans above $0.2 \mathrm{meV}$. However, for

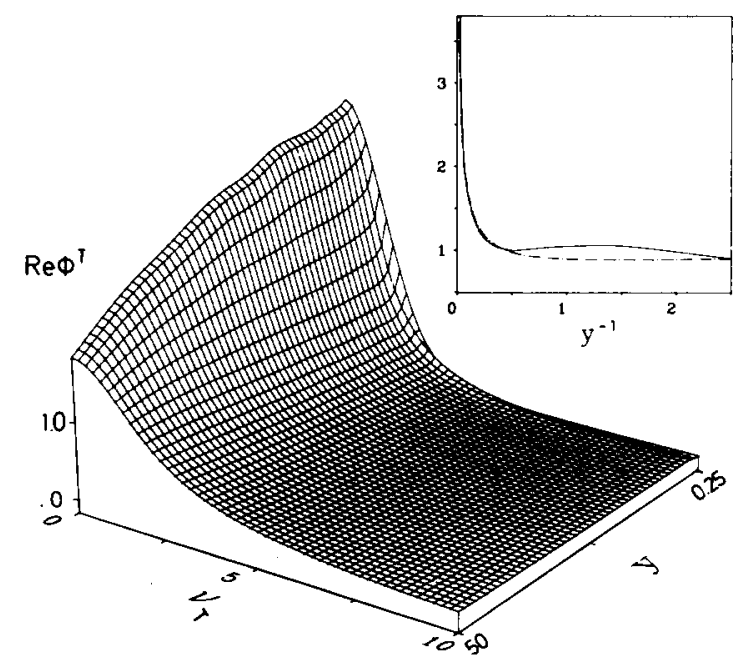

Fig. 4. Real part of the scaling function for the transverse Kubo relaxation function $\phi^{\mathrm{T}}\left(x=0, y, \nu_{\mathrm{T}}\right)$ versus $y=q_{\mathrm{D}} / q$ and $\nu_{\mathrm{T}}$ at $T=T_{\mathrm{c}}$. Inset: Linewidth of the transverse scaling function versus $y^{-1}=q / q_{\mathrm{D}}$ at $T_{\mathrm{c}}$. Solid line: half width at half maximum of the complete solution of the MC equations; dash-dotted line: lorentzian approximation.

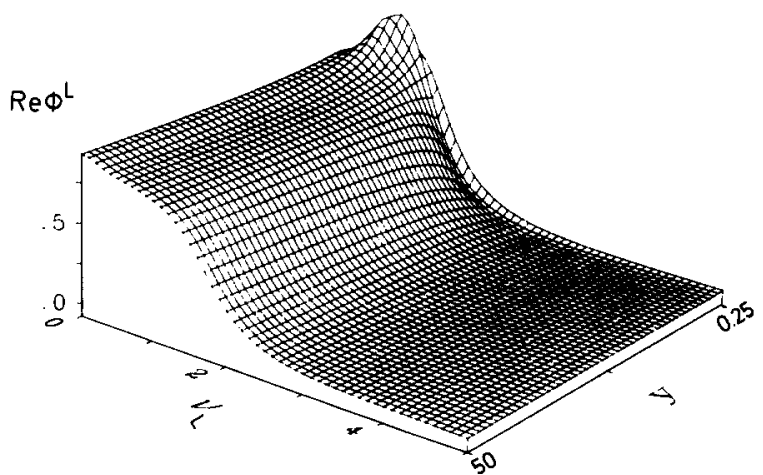

Fig. 5. Real part of the scaling function for the longitudinal Kubo relaxation function $\phi_{\mathrm{L}}\left(x=0, y, \nu_{\mathrm{L}}\right)$ versus $y=q_{\mathrm{D}} / q$ and $\nu_{\mathrm{L}}$ at $T=T_{\mathrm{c}}$. 


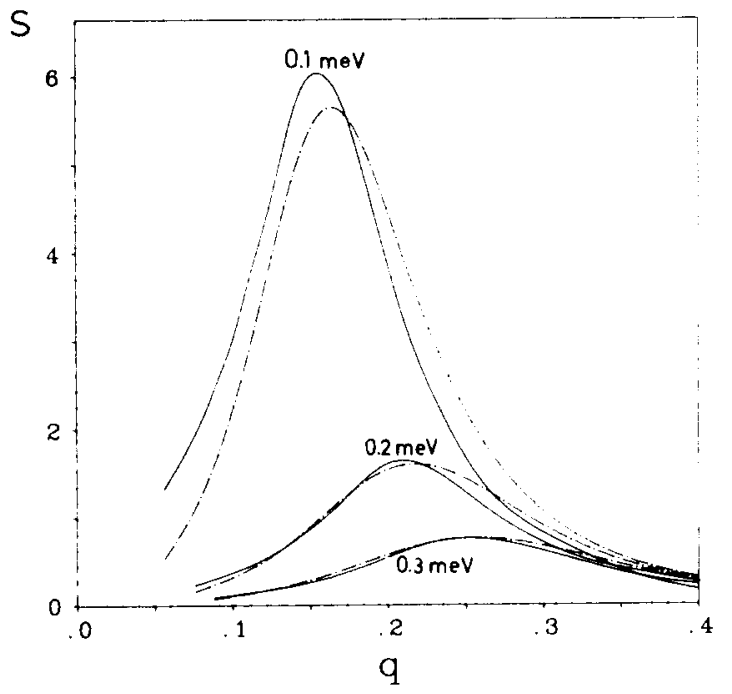

Fig. 6. Constant- $E$ scans at $T_{\mathrm{c}}$ for energies: $E=0.1 \mathrm{meV}, 0.2 \mathrm{meV}$ and $0.3 \mathrm{meV}$ versus wavevector $q$ in $\AA^{-1}$. Solid line: MC theory including dipolar interactions; dash-dotted line: RG theory without dipolar interactions (eqs. (40), (41) of ref. [20]).

lower energies the dipolar interaction shifts the peak positions to smaller wavevectors with respect to the RG results. This is exhibited in fig. 6 , where we compare our $\mathrm{MC}$ results for dipolar ferromagnets with $\mathrm{RG}$ results (see eqs. (40) and (41) in ref. [20]), which include exchange forces only. Further experiments at lower energies are needed to test this prediction.

Recently, in constant energy scans above $T_{\mathrm{c}}$ [27] significant disagreement was found with the RG results of ref. [19]. We expect that this failure of isotropic scaling may be remedied naturally within our MC theory. This requires the solution of the $\mathrm{MC}$ equations (15), (16) above $T_{\mathrm{c}}$ which is in progress.

For the numerical solution of the MC equations it was convenient to scale the frequencies with linewidths obtained in the lorentzian approximation. Of course from the final result the exact half width at half maximum (HWHM) of the relaxation functions can be obtained. In the inset of fig. 4 we compare these two characteristic frequencies for the transverse Kubo relaxation function $\phi^{\mathrm{T}}(x=0, y, \omega)$ at $T_{\mathrm{c}}$, where both functions are normalized to 1 for $y^{-1} \gg 1$. This leads for the lorentzian width to an effective non-universal constant $A_{\text {eff }}=7.1$ $\mathrm{meV} \AA^{5 / 2}$ and for the HWHM to a value $A_{\text {eff }}=8.5 \mathrm{meV} \AA^{5 / 2}$. The latter is quite close to the experimentally fitted values $8.3 \mathrm{meV} \AA^{5 / 2}[26]$ and $8.7 \mathrm{meV} \AA^{5 / 2}$ [2]. Taking into account this enhancement of the nonuniversal constant $A$ by a factor 1.2 we find $A_{\text {eff }}=128.6 \mathrm{meV} \AA^{5 / 2}$ for $\mathrm{Fe}$, which is also quite close to the experimental value $130 \mathrm{meV} \AA^{5 / 2}$ [2]. Except for this change of the non-universal scale there are only minor differences between the lorentzian width and the HWHM, which makes clear why the lorentzian approximation gives a good description for quantities depending on the linewidth only [12,13].

In conclusion we note that there is a crossover in the lineshape of the transverse Kubo function due to the additional dipolar interaction from a shape similar to that found in renormalization calculations at small $y$ (i.e. $q \geqslant q_{\mathrm{D}}$ ) to a lorentzian like shape at $q \ll q_{\mathrm{D}}$. The time dependence of the longitudinal shape function is gaussian for small times and shows an oscillatory behaviour. The lineshape crossover has only small effects on the crossover in the linewidth, which explains why the lorentzian approximation is excellent for the linewidth. Comparing our theory with Mezei's spin echo measurements, we have found that the MC theory accounts very well for the data. Especially the rapid drop off at small times is explained very naturally in contrast to theories taking into account solely the exchange interaction.

This work has been supported by the German Federal Minister for Research and Technology (BMFT) under contract number 03-SC1TUM-0. 


\section{References}

[1] F. Mezei, Phys. Rev. Lett. 49 (1982) 1096.

[2] F. Mezei, J. Magn. Magn. Mater. 45 (1984) 67.

[3] P. Böni and G. Shirane, Phys. Rev. B 33 (1986) 3012.

[4] P. Böni, G. Shirane, H.G. Bohn and W. Zinn, J. Appl. Phys. 61 (1987) 8.

[5] R.A. Dunlap and A.M. Gottlieb, Phys. Rev. B 22 (1980) 3422.

[6] C. Hohenemser, L. Chow and R.M. Suter, Phys. Rev. B 26 (1982) 5056.

[7] C. Hohenemser, R.M. Suter, L. Chow, M.A. Kobeissi, R. Dunlap and M.A. Gottlieb, Hyp. Int. 10 (1981) 887.

[8] J. Kötzler, W. Scheithe and R. Blickhan, Solid State Commun. 26 (1978) 641.

[9] J. Kötzler, G. Kamleiter and G. Weber, J. Phys. C 9 (1976) L361.

[10] J. Kötzler and H. von Philipsborn, Phys. Rev. Lett. 40 (1978) 790.

[11] J. Kötzler and W. Scheithe, J. Magn. Magn. Mater. 9 (1978) 4.

[12] E. Frey and F. Schwabl, Phys. Lett. A 123 (1987) 49.

[13] E. Frey and F. Schwabl, Z. Phys. B (1988), to be published.

[14] J. Hubbard, J. Phys. C 4 (1971) 53.

[15] F. Wegner, Z. Phys. 216 (1968) 433.

[16] S.W. Lovesey and R.D. Williams, J. Phys. C 19 (1986) L253.

[17] U. Balucani, M.G. Pini, P. Carra, S.W. Lovesey and V. Tognetti, J. Phys. C 20 (1987) 3953.

[18 ] R. Folk and H. Iro, Phys. Rev. B 32 (1985) 1880.

[19] R. Folk and H. Iro, Phys. Rev. B 34 (1986) 6571.

[20] H. Iro, Z. Phys. B 68 (1987) 485.

[21] V. Dohm, Solid State Commun. 20 (1976) 657.

[22] J.K. Bhattacharjee and R.A. Ferrell, Phys. Rev. B 24 (1981) 6480.

[23] F. Mezei, Physica B 136 (1986) 417.

[24] A. Aharony and M.E. Fisher, Phys. Rev. B 8 (1973) 3323.

[25] A.D. Bruce, J.M. Kosterlitz and D.R. Nelson, J. Phys. C 9 (1976) 825.

[26] P. Böni, M.E. Chen and G. Shirane, Phys. Rev. B 35 (1987) 8449.

[27] P. Böni, G. Shirane, H.G. Bohn and W. Zinn, preprint, Conf. on Magnetism, Chicago 1987 (BNL 40220). 